\title{
A method to enhance the accuracy of time of flight measurement systems
}

\author{
J.Papadoudis, A. Georgiadis, Leuphana Universität Lüneburg, C. Koch, Fachhochschule \\ Emden/Leer, St, Klein, Inosens GmbH, Dahlenburg (Germany) \\ Leuphana Universität Lüneburg \\ Volgershall 1 \\ 21339 Lüneburg
}

\begin{abstract}
A ToF-camera operates with modulated visible and near-infrared radiation, which is detected and demodulated simultaneously by a 2-D array of pixels. The phase shift between the sent signal and the received signal is an indicator for the object's distance. The amplitude of the radiation gives additional information for its reflectance. Unfortunately, measurements of distances performed with almost all cameras available on the market show very high deviations. The main reason for them seems to be the influence of stray light, which is detected by the camera with the modulated radiation. Another source of uncertainties is the low sampling rate along the received signal. In the first part of this paper, experiments using different cameras and alternating environments have been performed in order to determine the deviations, which are up to $20 \mathrm{~cm}$ over a distance of $150 \mathrm{~cm}$. A survey of existing cameras as well as their performance has taken place. In the second part of the present work two approaches for the eliminations of the deviations have been introduced. In the first approach spline functions have been calculated using reference measurements for distances between $5 \mathrm{~cm}$ and $200 \mathrm{~cm}$. Those functions are used for the calculation of the uncertainties of each pixel. Alternatively the splines can be replaced by a gain matrix which is multiplied with the image matrix in order to equalize the error of the distance measurement of the pixel. Subsequently only one spline is needed in order to adjust the distances. In this case the calculation is faster but it cannot be applied in every case. The reflectance of the objects in the scene has not been taken into account yet. The opposite happens in the second approach in order to enhance the accuracy of the measurement. This is done by estimating the reflectance of the nearest object in dependency of the measured amplitude and distance. The reflectance of the background, respectively everything behind the nearest object, is estimated by using the measured amplitude of the background and the distance of the nearest object and the background. By using both reflectance factors and the distances as indicators for further smoothing functions the error of measurement due to the reflectance can be determined. After applying both methods the error of measurement decreased in our tests from about $20 \mathrm{~cm}$ to $3 \mathrm{~cm}$.
\end{abstract}

\section{Measuring principle}

A Time-of-Flight (ToF) camera consists of a CCD/CMOS-Chip and an array of LEDs transmitting visible or near-infrared radiation. The transmitted signal illuminates the scene and the reflected signal is received by the CCD/CMOS-Chip. Due to the covered distance $d$ of the received signal there is a phase shift between the transmitted signal, with the frequency $f$, and the received signal. The phase shift, directly related to the distance, can be converted into a distance.

$$
\varphi=\frac{4 \pi f}{c} d
$$

The amplitude of the received signal diversifies based on the surface of the objects in the scene. Hence the amplitude image equals the intensity image. The phase and amplitude can not be measured directly from the received signal therefor it is necessary to correlate the signal with an electrical reference signal. The received signal $s(t)$ and the reference signal $g(t)$ are sampled at 4 different phase positions, e.g $\alpha_{n}=\left\{0, \frac{\pi}{2}, \pi, \frac{3}{2} \pi\right\}$. The signals are multiplied and the integration of the product equals the value of the correlation-function (CF) at the specific phase position.

$$
C F\left(\alpha_{n}\right)=\int_{0}^{2 \pi}\left(s\left(\alpha_{n}\right) * g\left(\alpha_{n}\right)\right)
$$

The values of the correlation function can be used to determine the phase shift through: 


$$
\varphi=\tan ^{-1}\left(\frac{C F\left(\alpha_{4}\right)-C F\left(\alpha_{2}\right)}{C F\left(\alpha_{1}\right)-C F\left(\alpha_{3}\right)}\right)
$$

Or respectively the amplitude through:

$$
a=\frac{\sqrt{\left|C F\left(\alpha_{4}\right)-C F\left(\alpha_{2}\right)\right|^{2}-\left|C F\left(\alpha_{1}\right)-C F\left(\alpha_{3}\right)\right|^{2}}}{2}
$$

The following picture illustrates the equations above. The received signal is green and the reference signal blue. The gray area represents the integral.
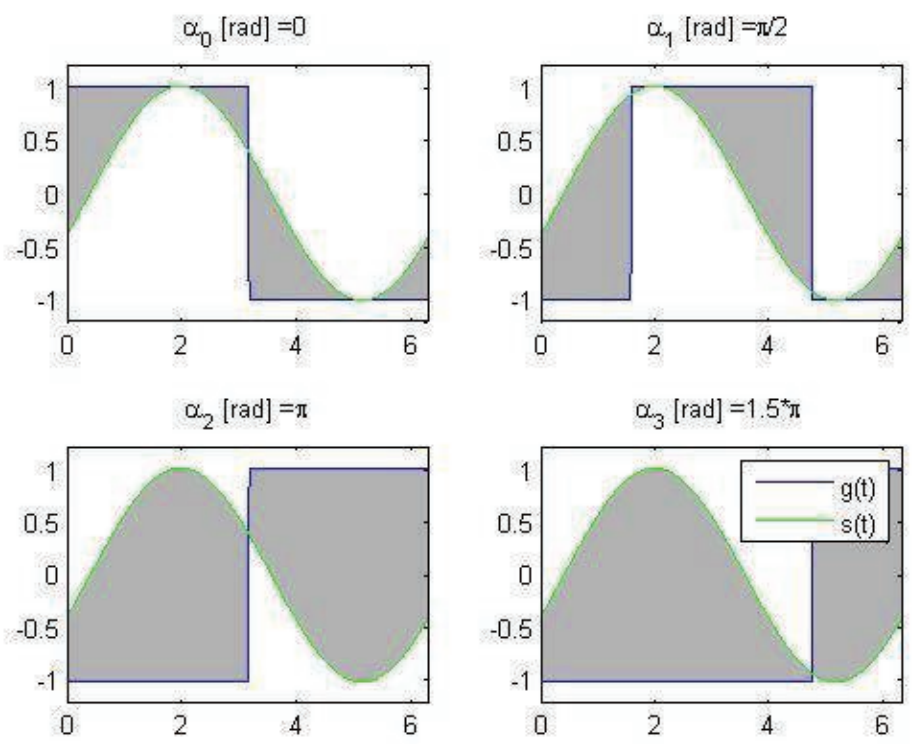

Figure 1.1: The integral of the received signal (green) multiplied with the reference signal (blue) represents the value of the correlation function at a given phase shift.

Figure 1.2 shows the points of the correlation function as well as the function itself.

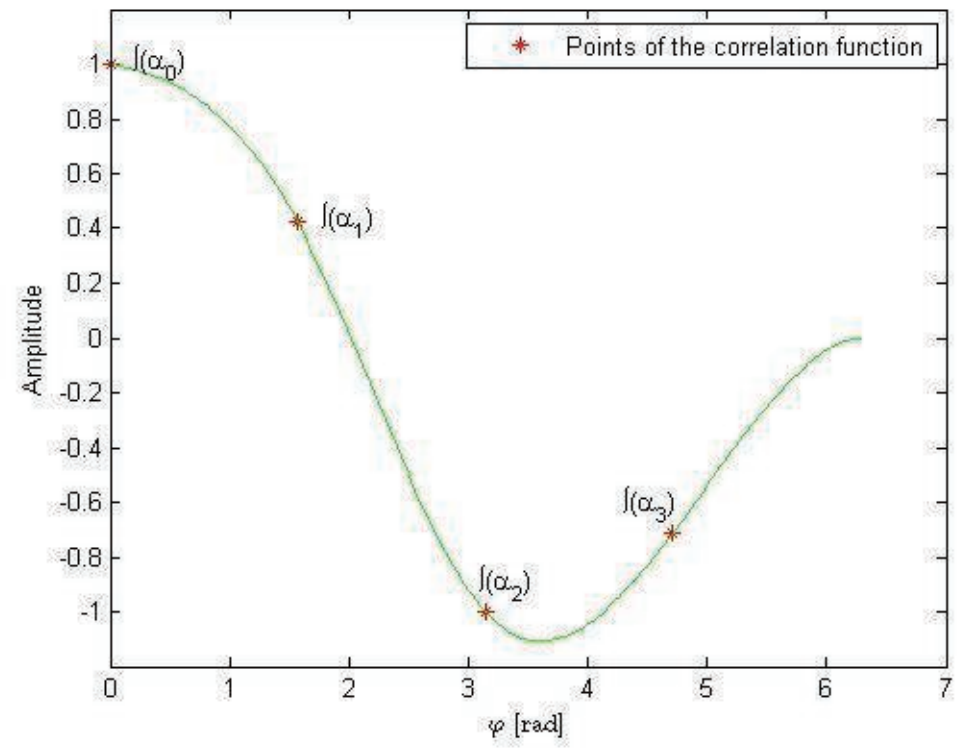

Figure 1.2: This figure shows the points of the correlation function obtained through equations $1.2 \mathrm{ff}$ 


\section{Benchmarking}

In the course of determining the errors of measurement six different 3D Sensors haven been evaluated. The sensors were compared in respect to:

- $\quad$ Temperature drift

- Moving objects

- Stray light

- Complex scenes

This paper focus on the results of the measuring inaccuracies due to complex scenes and stray light since the introduced methods in order to enhance the accuracy adresse these errors. In the following the detailed results of the "PMD[vision] O3" will be shown exemplariliy.

To verify the preciseness of the measured distance several pictures were made of a plane area at a determined distance. In addition two different complex scenes, shown in Figure 2.1, were investigated.

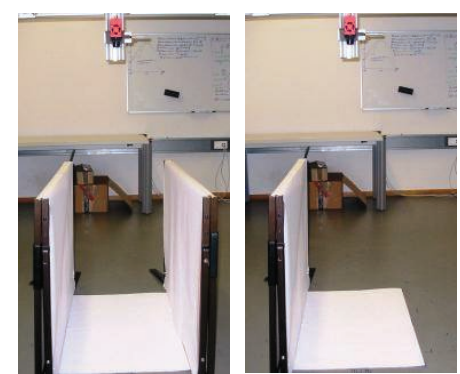

Figure 2.1: The left picture shows the closed scene and the right the open scene.

The experiments show a low distance measurement precision due to its low optical power among other things. Figure 2.2 illustrate the measurement of a plane area at the distance of $700 \mathrm{~mm}$. The camera specifies the distance about $950 \mathrm{~mm}-1000 \mathrm{~mm}$. In addition the area seems curved. The impact of stray light was measured by adding an object (Target) into the scene. The background was a wall. On the right side of Figure 2.2 the measured distance and the real distance is shown. It is quite obvious that the stray light results in an error of measurement especially for the background (wall).

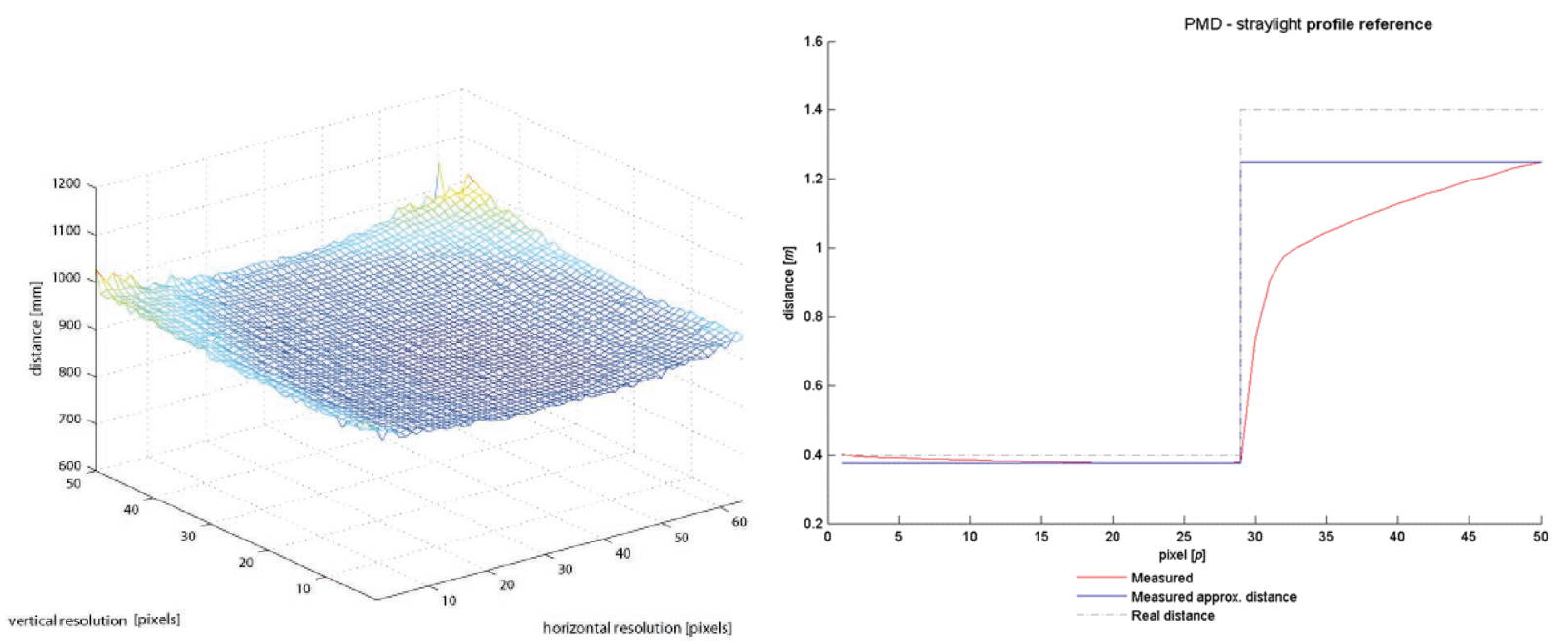

Figure 2.2: This figure shows the errors of measurement of a plane are (left) and the impact of stray light (right).

\section{Errors of measurement}

The errors of measurement due to the curvature and the impact of the stray light shown in chapter 2, will be explained in the following. 


\subsection{General offset}

As described, the distance is measured with the aid of a correlation function, by using four sampling points. Supposing that the received signal is unaffected by external parameters, such as insufficient optical power, the quality of the optics, etc., four sampling points would be enough to determine the correlation function, referring to the distance, exactly. Since the received signal is affected by the optics, the scene itself and the construction of the camera, each camera has a different error of measurement, so it is necessary to compensate those errors. Figure 2.2 (left) shows an exposure of a plane area at the distance of $700 \mathrm{~mm}$. A spline $s:[a, b] \rightarrow \mathbb{R}$ can be determined by evaluating a series of exposures at the distances $a$ to $b$ with a small step size. The difference between the measured distance and the real distance is the result of the spline with the measured distance as input. So let $d_{m}$ be the measured distance and $e$ the error of measurement the spline is defined as: $e=s\left(d_{m}\right)$. It is obvious that a single spline cannot remove the error of each pixel $P$ so the spline in order to correct the whole image must differ with each pixel. Thus the final spline can be defined as follows:

$$
e=e(P)=s\left(d_{m}, P\right)
$$

Since this adjustment assures that every single pixel is treated separately it is very precisely but also computationally intensive.

Let $/$ be the $\mathrm{n} \times \mathrm{m}$ image-matrix and $\mathrm{A}$, independent of the measured distance $d$, be an $\mathrm{n} \times \mathrm{m}$ matrix and $\mathrm{B}$ the result matrix, defined as follows:

$$
\left(b_{r p}\right)=\left(i_{r p}\right) *\left(a_{r p}\right)
$$

with

$$
\sum_{r=1}^{n} \sum_{p=1}^{m}\left|\left(b_{r p}\right)-k\right|=0
$$

with $k \in \mathbb{R}$ changing over the distance.

After defining the matrix $A$, the matrix $B$ has to be calculated for each distance. Afterwards the spline $s\left(d_{m}\right)=k$ can be defined such that $B+k=d$. The adjusted image $I_{a}$ can be evaluated as follows:

$$
I_{a}=I * A+s\left(d_{m}\right)
$$

The described method ignores the impact of stray light. Scenes with objects evoke stray light which causes an additional error, which can be eliminated with the method introduced in the following section.

\subsection{Stray light}

Stray light will be defined in this paper as "unwanted reflections caused by the scene". In order to investigate the impact of stray light, several exposures of a scene with one object in front of plane, were made, with alternating distances and reflectances of the objects. More complex scenes can be reduced successively to this measuring setup.

Tests showed that the error of measurement in consequent of stray light is dependent on:

- The distance of the background

- The distance of the object

- The reflectance of the object

- The reflectance of the background

A method has been designed which eliminates the error caused only by stray light.

The algorithm bases on reference exposures with alternating distances of the background and the object as well as with alternating reflectances. The measured values of the amplitudes of the background and the object were indexed in respect to their distance, reflectance and separated whether this values belong to the background or the object. At first the reflectances of the object and the background has to be calculated. Depending on the reference exposures a regression spline $s$ of the type:

$$
R=s\left(d_{o}, d_{b}, a_{o}, a_{b}\right)
$$


where $d_{o}, d_{b}$ representing the measured distances of the object and the background and $a_{o}, a_{b}$ the lowest value of the amplitude in the region specified as object respectively background, is determined. The spline is calculated separately for the background and the object. The result $R$ is an indicator for the reflectance of the object or the background with $0 \leq R \leq 1$ where the highest reflectance of the reference exposures is defined as 1 .

With the derived reflectances the error of the distances can be calculated. Let $M$ be a family of sets of the measured distance in dependency of the reflectance of the object and the background. This set can be visualized as seen in Figure 3.1.

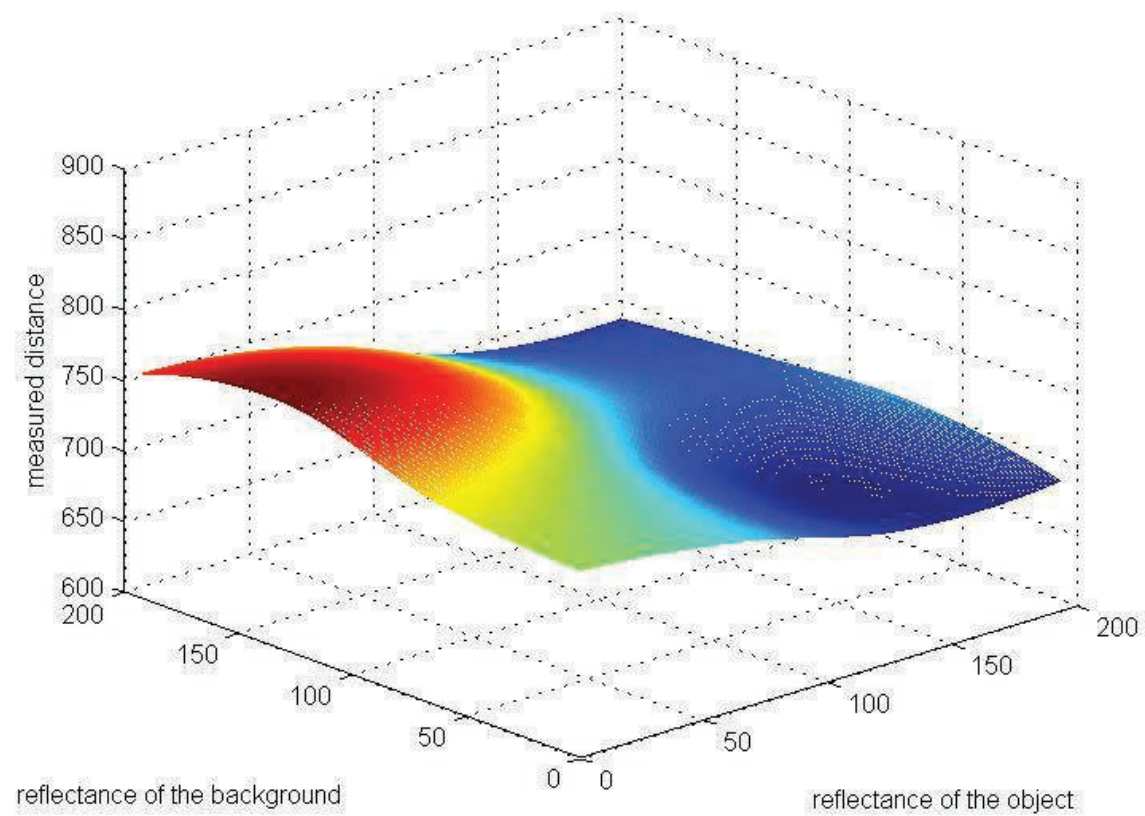

Figure 3.1: This plot shows the measured distance dependent on the reflectances of the object and the background

Each element in the set $M_{i}$ can be indexed in respect to the reflectance of the object and the reflectance of the background determined in the previous step. These indexes are $n$ for the object's distance and $m$ for the background's reflectance. $M$ is the family of sets $M_{i}$ where the index $i$ is an indicator for a combination of the real distance of the background and the object of the reference exposure. Based on the calculated reflectances a new set $P=\left\{M(n, m)_{i} \mid n, m\right.$ the refelctance - indicator and $\left.\forall i\right\}$ is created. This set represents the checkpoints for a smoothing function $P_{s}$. Let $d_{a}$ be the measured distance without the influence of stray light evaluated through $P_{s}\left(d_{a}\right)=d_{o}$ respectively $P_{s}\left(d_{a}\right)=d_{b}$. Thus the error of measurement $e$ can be calculated by $e=d_{a}-d_{o}$ and the image can be adjusted.

After removing the stray light with this algorithm, the method explained in section 3.1 can be used to eliminate the remaining error of measurement.

\section{Results}

The introduced methods are able to reduce the error of measurement from about $20 \mathrm{~cm}$ to $3 \mathrm{~cm}$. The method shown in section 3.1 reduces the general offset and the curvature of the image. Figure 4.1 shows a result for a distance adjusted image, with no stray light.

The method causes a significant improvement of the measurement. Table 4.1 shows the deviation of some measurements between $700 \mathrm{~mm}$ and $880 \mathrm{~mm}$. 


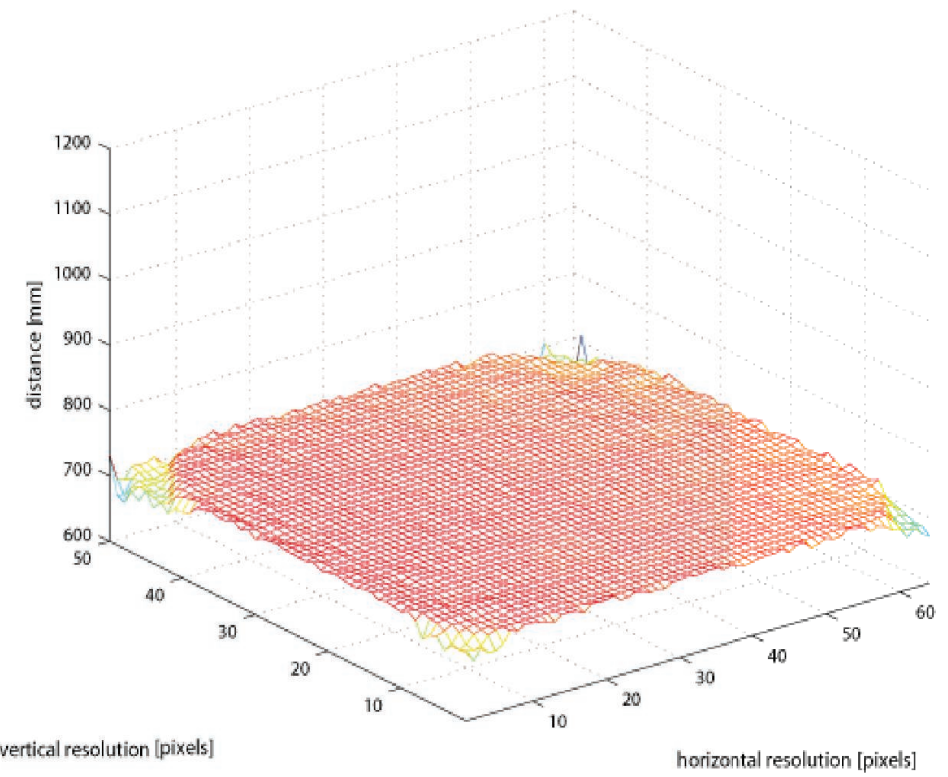

Figure 4.1: This figure shows the adjusted image of a plane area at the distance of $700 \mathrm{~mm}$.

\begin{tabular}{rrr}
\hline $\mathbf{d}_{\mathbf{r}}[\mathbf{m m}]$ & $\mathbf{d}_{\mathbf{m}}[\mathbf{m m}]$ & $\Delta d[\mathbf{m m}]$ \\
\hline 700 & 910.7 & 210.7 \\
720 & 927.1 & 207.1 \\
740 & 950.8 & 210.8 \\
760 & 961.1 & 201.1 \\
780 & 978.8 & 198.8 \\
800 & 999.1 & 199.1 \\
820 & 1016.1 & 196.1 \\
840 & 1033.4 & 193.4 \\
860 & 1055.4 & 195.4 \\
880 & 1070.7 & 190.7 \\
\hline
\end{tabular}

Table 4.1: This table shows the real distance $\left(d_{r}\right)$ the measured distance $\left(d_{m}\right)$ and the difference for several measurement.

After applying both algorithms on scenes affected with stray light, the error has been reduced to a mean of $32.6 \mathrm{~mm}$ and the standard deviation has been reduced from $12.39 \mathrm{~mm}$ to $8.47 \mathrm{~mm}$. So applying the algorithms on complex scenes improves the measurement significantly. The applied algorithm to compensate the error of measurement caused by stray light reduces the error about $70 \%$ compared to images only calibrated with in terms of offset and curvature.

\section{References}

Klein, S. (2010). Methode zur Kompensierung des Streulichtanteils bei der kontinuierlichen Modulationsinterferometrie. Dissertation Leuphana Universität Lüneburg: Sierke Verlag.

Koch, C. (2008). Messen und Klassifizieren mit Time-of-Flight Sensorik: Potential und Limitationen. PMD[vision] Day.

Kolb, A., \& Lindner, M. (2007). Calibration of the Intensity-Related Distance Error of the PMD TOF Camera.

Lange, R. (2000). 3D Time-of-Flight Distance Measurement with Custom Solid-State Image Sensors in CMOS/CCD-Technology. Siegen.

Papadoudis, J. (August 2009). Entwicklung eines Kalibrierverfahrens für dreidimensionale, phasenbasierte Lichtlaufzeit-Messsysteme. Bachelorarbeit Leuphana Universität Lüneburg.

Rapp, H. (2007). Experimental and Theoretical Investigation of Correlating TOF-Camera Systems. Heidelberg. 